\title{
Anomalous Isotopic Effect on the Lattice Parameter of Silicon
}

\author{
H.-C. Wille, Yu. V. Shvyd'ko, ${ }^{*}$ E. Gerdau, M. Lerche, M. Lucht, and H. D. Rüter \\ Institut für Experimentalphysik, Universität Hamburg, D-22761 Hamburg, Germany
}

\author{
J. Zegenhagen \\ European Synchrotron Radiation Facility, F-38043 Grenoble Cedex, France
}

(Received 19 June 2002; published 27 December 2002)

\begin{abstract}
The difference $\Delta a=a_{(30)}-a_{(28)}$ of the lattice parameter of ${ }^{30} \mathrm{Si}$ and ${ }^{28} \mathrm{Si}$ crystals is measured over a temperature range from 4.7 to $700 \mathrm{~K}$. In disagreement with existing knowledge, the strongest isotopic effect is not detected at the lowest achieved temperature $T=4.7 \mathrm{~K}$. An anomalous behavior is observed: The relative difference $|\Delta a / a|$ attains its maximum value of 56.8(5) ppm at $T=$ $75(10) \mathrm{K}$. The anomalous behavior is attributed to the influence of phonon modes with negative Grüneisen parameters. At $T=700 \mathrm{~K}$ the effect still amounts to $30 \%$ of the maximal value. The experimental data are consistent with an approach based on the density-functional perturbation theory.
\end{abstract}

DOI: $10.1103 /$ PhysRevLett.89.285901

The lattice parameter of crystals composed of different isotopes of the same element may be different. This statement was put forward by London in 1958 [1]. It is a quantum mechanical effect due to the anharmonicity of the potential and the different zero point motion of isotopes with different masses. It is more pronounced for light elements where the relative mass difference of the isotopes is greater. Assuming the zero point motion to be the dominating mechanism, one expects the strongest isotopic effect at $T=0 \mathrm{~K}$.

The isotopic effect was studied experimentally in different crystals. In diamond, a decrease of the lattice parameter was observed with increasing concentration of ${ }^{13} \mathrm{C}$ [2]. The temperature dependence of the effect was investigated for $\mathrm{Ge}$ and $\mathrm{Si}$ crystals between 30 and $300 \mathrm{~K}$ $[3,4]$. In these studies, the greatest difference in lattice parameters was observed at the lowest achieved temperature. Silicon is of special interest, because it could be used for an accurate determination of the Avogadro constant and a redefinition of the SI unit of mass [5]. The isotopic effect on the lattice parameter of silicon crystals was investigated in numerous theoretical papers [6-8]. However, they give significantly different predictions on the magnitude of the effect. Furthermore, some of them predict the greatest difference in the lattice parameters at a temperature well above $0 \mathrm{~K}$. This behavior has not yet been proven. Also the evolution of the effect above $300 \mathrm{~K}$ has not been investigated experimentally so far.

The purpose of the present Letter is to investigate the isotopic effect on the lattice parameter of silicon in a wide temperature range and with a precision that is sufficient to confirm or discard existing theoretical models. At low temperatures the variation of the lattice parameter difference is small. To measure the temperature dependence reliably, a technique with a relative resolution of about $1 \mathrm{ppm}$ is required.

Bragg backscattering of highly monochromatic synchrotron radiation can be used for direct and precise mea-
PACS numbers: 65.40.-b, 06.30.Dr, 61.10.Eq, 91.65.Dt

surements of lattice parameters. Recently, it was used to introduce a $\gamma$-ray wavelength standard for atomic scales [9], for the determination of the wavelengths of Mössbauer radiation and the temperature dependence of the lattice parameter of sapphire [10]. The outstanding feature of Bragg backscattering is its narrow spectral bandwidth combined with the large angular acceptance [11]. Atomic planes of a crystal with interplanar distance $d$ reflect $\mathrm{x}$ rays in back direction at the Bragg energy $E_{0}=$ $h c / 2 d$ (small refraction effects are neglected). Here $h$ is the Planck constant, and $c$ is the velocity of light in vacuum. If the interplanar distance changes by $\delta d$ the Bragg energy changes by $\delta E_{0}=-E_{0} \delta d / d$. This simple relation underlies the experimental technique: by measuring the relative change of the Bragg energy, one can determine directly the relative change of the interplanar distance. The relative energy width of Bragg back reflections in silicon crystals is $\Delta E / E_{0} \leq 1 \mathrm{ppm}$ at energies $E_{0} \geq 10 \mathrm{keV}$. Thus the use of Bragg back diffraction in silicon with Bragg energies $E_{0} \geq 10 \mathrm{keV}$ may allow one to resolve relative variations of the interplanar distance $\delta d / d$ as small as $1 \mathrm{ppm}$. To implement this technique also the synchrotron radiation has to be monochromatized to a relative energy bandwidth of about $1 \mathrm{ppm}$. This is achieved with the help of high-energy-resolution monochromators designed for nuclear resonant scattering experiments (see [12] for a review). The angular acceptance $\Delta \theta$ of Bragg back reflections in silicon is at least 1 order of magnitude larger than the divergence of beams available at modern synchrotron radiation facilities. Therefore, such measurements in backscattering geometry are not influenced by the beam divergence. We made use of the (1240) Bragg reflection in silicon with $E_{0}=14.438 \mathrm{keV}, \Delta E=6.2 \mathrm{meV}, \Delta \theta=0.9 \mathrm{mrad}$, and $\Delta E / E_{0}=0.43 \mathrm{ppm}$.

We have studied an epitaxial silicon crystal system consisting of a $550 \mu \mathrm{m}$ thick single crystal substrate of natural silicon $\left(92.2 \%{ }^{28} \mathrm{Si}, 4.7 \%{ }^{29} \mathrm{Si}\right.$, and $\left.3.1 \%{ }^{30} \mathrm{Si}\right)$ with 
about an $L_{\mathrm{e}}=15 \mu \mathrm{m}$ thick epitaxial single crystal layer of ${ }^{30} \mathrm{Si}$ on top. The contact plane is parallel to the (111) lattice planes. The mean average mass of natural silicon is $\bar{M}_{\mathrm{Si}}=28.086 \mathrm{amu}$. Because of the small deviation in their masses, ${ }^{28} \mathrm{Si}$ and natural $\mathrm{Si}$ are used synonymously in the following.

The growth of the epitaxial layer of ${ }^{30} \mathrm{Si}$ is performed at a temperature above $1000 \mathrm{~K}$ where its lattice mismatch with the substrate is close to zero. The film grows perfectly pseudomorphic and stays that way upon cooling down. However, the lattice mismatch increases with decreasing temperature, resulting in strain, which is enhanced by $\epsilon=1.43$ in the direction normal to the (111) plane, because the film is forced to stay coherent with the substrate in plane [13]. The relative difference in the lattice plane spacing $\Delta d / d=\left(d_{(30)}-d_{(28)}\right) / d_{(28)}$ for the (12 40 ) planes with an angle $\alpha=43.09^{\circ}$ to (111) can be related to the relative difference in the lattice parameter $\Delta a / a=\left(a_{(30)}-a_{(28)}\right) / a_{(28)}$ as $\quad \Delta a / a=(\Delta d / d) /$ $\left(\epsilon \cos ^{2} \alpha\right)$, cf. $[3,4]$.

The measurements were performed at HASYLAB (DESY), Hamburg, at the wiggler beam line BW4 and at the undulator beam line PETRA I. A schematic view of the experimental setup is shown in Fig. 1.

A high-heat-load monochromator $(\mathrm{M})$ provides radiation with about $2 \mathrm{eV}$ bandwidth at around $14.4 \mathrm{keV}$. The high-energy-resolution monochromator (HRM) reduces the bandwidth to $2 \mathrm{meV}$ ( $7 \mathrm{meV}$ ) at PETRA I (BW4) and is tunable with sub-meV steps over a range of at least $160 \mathrm{eV}$. The detector (D) is a semitransparent avalanche photodiode with $1 \mathrm{~ns}$ time resolution. The signals from the incident and backreflected photons are distinguished due to their time of flight difference. The sample is placed in an oven or in a cryostat depending on the desired temperature range. A computer controlled temperature stabilization keeps the temperature stable within a few $\mathrm{mK}$ [14]. To avoid multiple beam scattering in $\mathrm{Si}$ [15-17] the measurements are performed about $1 \mathrm{mrad}$ off exact normal incidence to the (1240) planes.

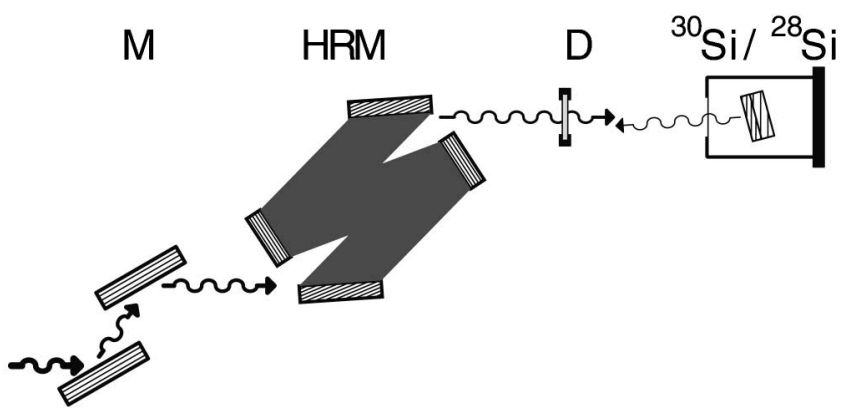

FIG. 1. Schematic view of the experimental setup. M: high heat load monochromator; HRM: high-energy-resolution monochromator; D: semitransparent detector with 1 ns time resolution; ${ }^{30} \mathrm{Si} /{ }^{28} \mathrm{Si}: 15 \mu \mathrm{m}{ }^{30} \mathrm{Si}$ epitaxial layer on a substrate of $550 \mu \mathrm{m}$ natural $\mathrm{Si}$.
Figure 2 shows examples of energy spectra of Bragg backscattering from the layered silicon crystal system measured at two different temperatures $T_{\mathrm{a}}=320 \mathrm{~K}$ and $T_{\mathrm{b}}=678 \mathrm{~K}$. The sharp peak at $E=E_{0_{(28)}}$ is due to the reflection from the single crystal substrate of ${ }^{28} \mathrm{Si}$. $E_{0_{(28)}}$ changes with temperature. From the difference $\delta E \stackrel{20}{=}$ $E_{0_{(28)}}\left(T_{\mathrm{a}}\right)-E_{0_{(28)}}\left(T_{\mathrm{b}}\right)$ the relative temperature change in the lattice plane spacing in the single crystal substrate can be calculated as $\delta d_{(28)} / d_{(28)}=-\delta E / E_{0_{(28)}}$.

The broader peak at $E_{0_{(30)}}$ with superimposed oscillations on the wings is due to the reflection from the epitaxial layer of ${ }^{30} \mathrm{Si}$. The fact that $E_{0_{(30)}}>E_{0_{(28)}}$ directly shows that the lattice parameter of ${ }^{30} \mathrm{Si}$ is smaller than that of ${ }^{28} \mathrm{Si}$. The relative difference $\Delta d / d=\left[d_{(30)}(T)-\right.$ $\left.d_{(28)}(T)\right] / d_{(28)}(T)$ in lattice spacings can be calculated as $\Delta d / d=\left[E_{0_{(28)}}(T)-E_{0_{(30)}}(T)\right] / E_{0_{(28)}}$. The oscillations result from the interference of the waves reflected from the front and rear surface of the thin epitaxial layer. The period of oscillations is $h c / 2 L_{\mathrm{e}}$ (see, e.g., [17]).

The solid lines are fits using the dynamical theory of $\mathrm{x}$-ray diffraction in layered crystal systems at normal incidence $[17,18]$. The experimentally observed interference fringes are damped because of some fluctuations in the thickness $L_{\mathrm{e}}$ of the epitaxial layer. This is, however, without influence on the accuracy of the results. The relative difference $\Delta d / d$ is determined from the fits with an uncertainty of $\simeq 0.5 \mathrm{ppm}$.

Figure 3 shows the evaluated temperature dependence of the relative difference $\Delta a / a=\left(a_{(30)}-a_{(28)}\right) / a_{(28)}$ of the lattice parameters of ${ }^{30} \mathrm{Si}$ and ${ }^{28} \mathrm{Si}$. Figure 4 shows the change of each lattice parameter independently. By using the harmonic scaling law $T^{\prime}=T \sqrt{M_{(30)} / M_{(28)}}$ [20] the

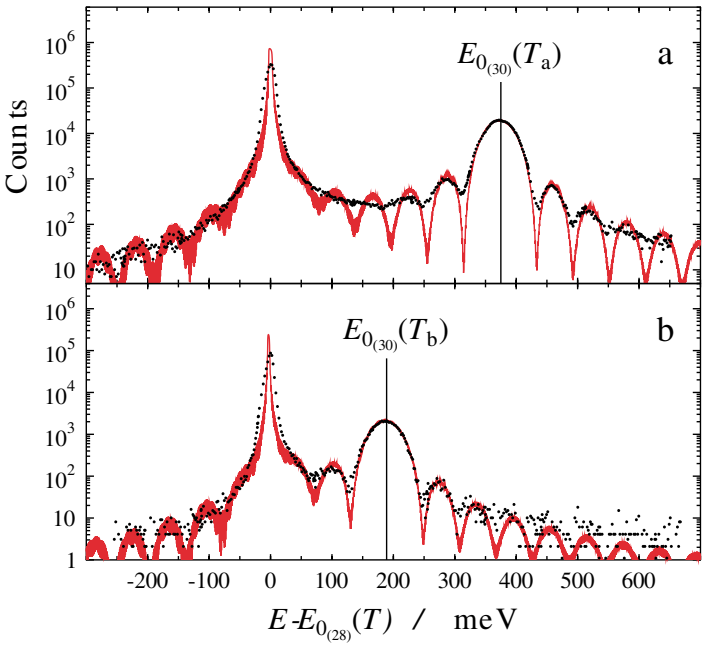

FIG. 2 (color online). Energy spectra of the (1240) Bragg back reflection from the layered epitaxial ${ }^{30} \mathrm{Si}$ and ${ }^{28} \mathrm{Si}$ crystal systems at different temperatures (a) $T_{\mathrm{a}}=320 \mathrm{~K}$ and (b) $T_{\mathrm{b}}=$ 678 K. $E_{0_{(28)}}\left(T_{\mathrm{a}}\right)-E_{0_{(28)}}\left(T_{\mathrm{b}}\right)=18.09(8) \mathrm{eV}$. The solid lines are fits with the dynamical theory of x-ray diffraction. 


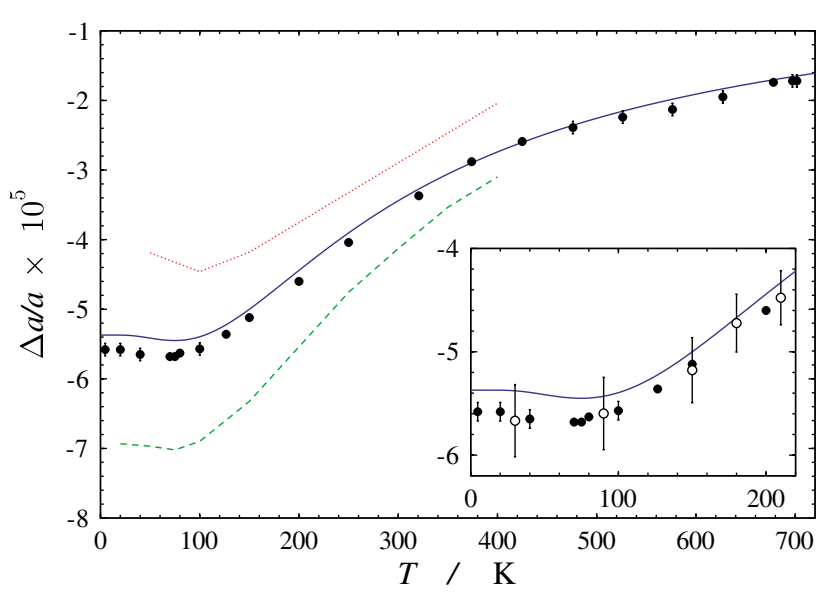

FIG. 3 (color online). Relative difference $\Delta a / a=$ $\left(a_{(30)}-a_{(28)}\right) / a_{(28)}$ of the lattice parameters in ${ }^{30} \mathrm{Si}$ and ${ }^{28} \mathrm{Si}$ vs temperature as determined from the (1240) Bragg backscattering spectra $(\bullet)$. The solid line shows predictions of Pavone and Baroni [8]; the dashed line shows calculations of Biernacki and Scheffler [7]; the dotted line is a guide to the eye for calculations of Herrero [6]. The open circles in the inset are recent experimental results of Sozontov et al. [4].

two dependences shown in Fig. 4 can be put one upon another so that $a_{(30)}(T) \simeq a_{(28)}\left(T^{\prime}\right)-\Delta a(0)$.

In the temperature range from 4.7 to $100 \mathrm{~K}$ the isotopic effect on the lattice parameter of silicon is strongest, but its variation with temperature is small (Fig. 3). The inset of Fig. 3 shows the low temperature region up to $220 \mathrm{~K}$. Here, a main result of the present study becomes obvious: the isotopic effect on the lattice parameter of silicon shows an anomalous behavior, as the absolute value $|\Delta a / a|$ of the relative difference of the lattice parameter of ${ }^{30} \mathrm{Si}$ and ${ }^{28} \mathrm{Si}$ first increases with temperature. It is maximal at 75(10) $\mathrm{K}$ and only then starts to decrease with further increasing temperature. The magnitude of the local maximum is $|\Delta a / a|=56.8(5) \mathrm{ppm}$. Even at $700 \mathrm{~K}$, well above the Debye temperature of silicon, the effect is still as large as $30 \%$ of the maximum value. The anomaly of the isotopic effect is to our knowledge an unreported experimental result. The recently published data of Sozontov et al. [4], shown in the inset of Fig. 3, are in agreement with our result. However, because of the larger error and the lack of data at about $75 \mathrm{~K}$ the anomalous effect could not have been detected in [4].

As will be explained in the following, the anomaly in the isotopic effect is closely related to the well known anomaly in the temperature dependence of the lattice parameter of $\mathrm{Si}[21,22]$ - the minimum at $125 \mathrm{~K}$ in Fig. 4. It is attributed to the role of the transversal acoustic phonon branches with negative Grüneisen parameter at low temperatures in $\mathrm{Si}$ [23]. However, since the minima in Fig. 4 and 3 are at different temperatures, the negative Grüneisen parameter cannot be alone responsible for the anomaly in the isotopic effect.

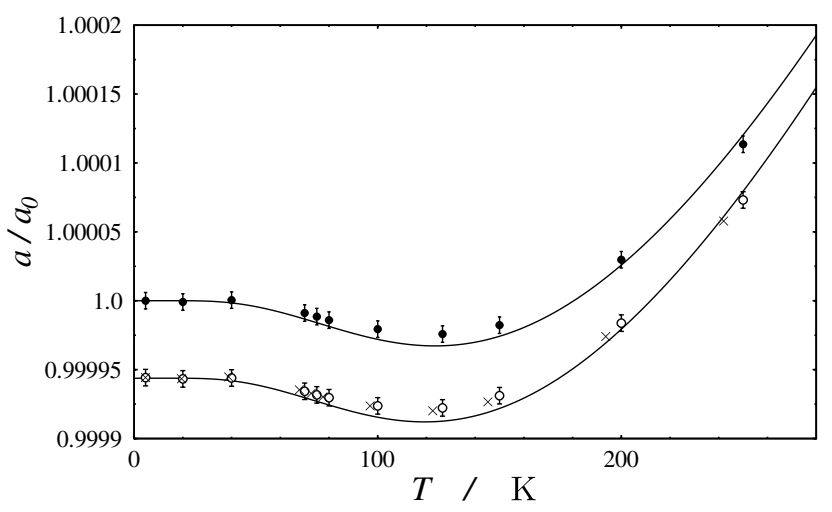

FIG. 4. Lattice parameters $a_{(28)}(\bullet)$ and $a_{(30)}$ ( ० ) vs temperature $T$ measured in the units of $a_{0}=a_{(28)}(4.7 \mathrm{~K})$. The solid lines are the calculations [19]. The crosses are generated by the transformation: $a_{(28)}\left(T^{\prime}\right)-\Delta a(0), T^{\prime}=T \sqrt{M_{(30)} / M_{(28)}}$.

Crystals with atoms of larger mass $M$ possess lower vibrational frequencies, as the latter scale with $1 / \sqrt{M}$. This is another fact essential for the qualitative explanation of the main experimental results. At $T=0$, the higher frequency of the lighter isotope $\left({ }^{28} \mathrm{Si}\right)$ results in a larger vibration amplitude and this together with the anharmonicity of atomic vibrations accounts for a larger lattice parameter. But why does the difference between the lattice parameters increase in one temperature region and decrease in another region? This can also be attributed to the aforementioned dependence of the phonon frequencies on the atomic mass. The lower the frequency, the more phonons can be excited at a given temperature, and thus a more rapid change of the lattice parameter with temperature should be observed for a crystal composed of a heavier isotope. In our particular case, the lattice parameter of ${ }^{30} \mathrm{Si}$ should change at a higher rate than that of ${ }^{28} \mathrm{Si}$. Still, the question remains: What is the sign of these changes? If the Grüneisen parameter is positive, then the lattice expands. In our particular case this means that under these conditions the ${ }^{30} \mathrm{Si}$ lattice expands faster than that of ${ }^{28} \mathrm{Si}$. Thus the lattice parameters in both crystals should converge with temperature. Such behavior is observed in our experiment at higher temperatures. However, if on the contrary, the Grüneisen parameter is negative, then the crystal lattice should contract. In our particular case, this means that the ${ }^{30} \mathrm{Si}$ lattice should contract faster than that of ${ }^{28} \mathrm{Si}$. Thus, the lattice parameters should diverge with temperature. Such behavior is observed at lower temperatures. If at this point, one remembers that just in silicon at low temperatures the phonon modes of the transversal acoustic branch with negative Grüneisen parameter dominate [23], then this fact completes the picture accounting for the main experimental observations.

Figure 3 demonstrates that the predictions of the theoretical approaches [6-8] are different. It is beyond the scope of this Letter to discuss them in detail. We give only 
a very brief account of the theoretical approaches focusing on their differences. Herrero's results [6] are based on Feynman path-integral calculations, Biernacki and Scheffler [7] and Pavone and Baroni [8] perform calculations in the quasiharmonic approximation using the density-functional theory. The crucial difference of the three approaches is the way the interatomic force constants and their volume dependence are calculated.

The potential applied by Herrero [6] is of the StillingerWeber type. The parameters of the potential are empirically chosen. It is reported that the applied model does not reproduce the negative thermal expansion coefficient of $\mathrm{Si}$, and the predicted lattice parameter values differ by about $0.2 \%$ from experimental results. Herrero gives an error range of about $6 \mathrm{ppm}$ on his results, which is not shown in Fig. 3. However, even within this error range, there is no agreement with our data.

Biernacki and Scheffler [7] apply first-principles pseudopotentials for the determination of the volume dependence of the free energy. Then the force constants are evaluated as an analytical expansion of the free energy in a Keating model representing central first neighbor and noncentral second neighbor interaction. It seems that the approximation is not sufficient for a quantitative description of the isotopic effect, as the results strongly differ from our experimental results.

Pavone and Baroni [8] perform ab initio calculations within the plane-wave pseudopotential method. In their approach the ground state energy calculation is repeated for several volumes with a small deviation to the equilibrium volume. At each volume the force constants are calculated using the so-called density-functional perturbation theory. The applied formalism nearly perfectly reproduces the phonon spectra of silicon and germanium [24]. The good agreement with our experimental results (Fig. 3) gives clear evidence that it is also a valid description of the isotopic effect on the lattice parameter.

In conclusion, we have measured the temperature dependence of the difference of the lattice parameters of ${ }^{28} \mathrm{Si}$ and ${ }^{30} \mathrm{Si}$ over the temperature range from 4.7 to $700 \mathrm{~K}$. The achieved uncertainty of the measurements of the relative difference $\Delta a / a$ is $0.5 \mathrm{ppm}$. We observe an anomalous behavior of the isotopic effect in silicon: The magnitude of the relative difference in lattice parameter first increases with temperature, reaches a maximum of $|\Delta a / a|=56.8(5) \mathrm{ppm}$ at $75(10) \mathrm{K}$, and only then decreases. At $T=700 \mathrm{~K}$, well above the Debye temperature of silicon, the effect is still large: about $30 \%$ of the maximal value. The anomalous behavior can be explained by the influence of phonon modes with negative Grüneisen parameters on the thermal expansion and the dependence of phonon frequencies on the isotopic mass. Our data are in excellent agreement with the theoretical predictions of Pavone and Baroni and therefore evidently support their density-functional perturbation approach.

We are grateful to $\mathrm{H}$. Franz for assistance during the measurements at PETRA I, to M. Komuna for growing the epitaxial ${ }^{30} \mathrm{Si}$ layer, and to P. Pavone for many helpful discussions on different aspects of lattice dynamic theory and for putting theoretical data at our disposal.

*Electronic address: Yuri.Shvydko@desy.de

[1] H. London, Z. Phys. Chem. Neue Folge 16, 302 (1958).

[2] H. Holloway, K. Hass, M. Tamor, T. Anthony, and W. Banholzer, Phys. Rev. B 44, 7123 (1991).

[3] A. Kazimirov, J. Zegenhagen, and M. Cardona, Science 282, 930 (1998).

[4] E. Sozontov, L. Cao, A. Kazimirov, V. Kohn, M. Konuma, M. Cardona, and J. Zegenhagen, Phys. Rev. Lett. 86, 5329 (2001).

[5] P. Becker, in Pioneering Ideas for the Physical and Chemical Sciences, edited by W. Fleischhacker and T. Schönfeld (Plenum Publishing, New York, 1997).

[6] C. Herrero, Solid State Commun. 110, 243 (1999).

[7] S. Biernacki and M. Scheffler, J. Phys. Condens. Matter 6, 4879 (1994).

[8] P. Pavone and S. Baroni, Solid State Commun. 90, 295 (1994).

[9] Yu. V. Shvyd'ko, M. Lerche, J. Jäschke, M. Lucht, E. Gerdau, M. Gerken, H. D.Rüter, and H.-C. Wille, Phys. Rev. Lett. 85, 495 (2000).

[10] Yu. V. Shvyd'ko, M. Lucht, E. Gerdau, M. Lerche, E. Alp, W. Sturhahn, J. Sutter, and T. Toellner, J. Synchrotron Radiat. 9, 17 (2002).

[11] K. Kohra and T. Matsushita, Z. Naturforsch. A 27, 484 (1972).

[12] T. Toellner, Hyperfine Interact. 125, 3 (2000).

[13] J. Hornstra and W. Bartels, J. Cryst. Growth 44, 513 (1978).

[14] M. Lucht, Diploma thesis, University of Hamburg, 1998.

[15] J. Sutter, E. Alp, M. Hu, P. Lee, H. Sinn, W. Sturhahn, and T. Toellner, Phys. Rev. B 63, 094111 (2001).

[16] Yu. V. Shvyd'ko and E. Gerdau, Hyperfine Interact. 123/ 124, 741 (1999).

[17] Yu. V. Shvyd'ko, Habilitationsschrift, Universität Hamburg, DESY, Hamburg, 2002 (DESY-Thesis-2002028, ISSN 1435-8085).

[18] V. Kohn, Y. Shvyd'ko, and E. Gerdau, Phys. Status Solidi (b) 221, 597 (2000).

[19] P. Pavone (private communication).

[20] A. Zhernov, Phys. Solid State 40, 1658 (1998).

[21] H. Ibach, Phys. Status Solidi 31, 625 (1969).

[22] K. Lyon, G. Salinger, and C. Swenson, J. Appl. Phys. 48, 865 (1977).

[23] C. Xu, C. Wang, C. Chan, and K. Ho, Phys. Rev. B 43, 5024 (1991).

[24] P. Giannozzi, S. Gironcoli, P. Pavone, and S. Baroni, Phys. Rev. B 43, 7231 (1991). 